\title{
Inhibition of C6 glioma in vivo by combination chemotherapy of implantation of polymer wafer and intracarotid perfusion of transferrin-decorated nanoparticles
}

\author{
LEI HAN $^{1-3 *}$, YU REN $^{4 *}$, LIXIA LONG $^{5}$, YUE ZHONG $^{1-3}$, CHANGHONG SHEN $^{1-3}$, \\ PEIYU PU ${ }^{1-3}, X^{-}$YUO $^{5}$ and CHUNSHENG KANG ${ }^{1-3}$
}

\begin{abstract}
${ }^{1}$ Department of Neurosurgery, Laboratory of Neuro-Oncology, Tianjin Medical University General Hospital, Tianjin 300052; ${ }^{2}$ Key Laboratory of Post-trauma Neuro-repair and Regeneration in Central Nervous System, Ministry of Education, Tianjin 300052; ${ }^{3}$ Tianjin Key Laboratory of Injuries, Variations and Regeneration of Nervous System, Tianjin 300052; ${ }^{4}$ Tianjin Research Center of Basic Medical Science, Tianjin Medical University, Tianjin 300070; ${ }^{5}$ School of Materials Science and Engineering, Tianjin University, Tianjin 300070, P.R. China
\end{abstract}

Received June 6, 2011; Accepted August 22, 2011

DOI: $10.3892 /$ or.2011.1459

\begin{abstract}
The objective of this study was to develop a combination chemotherapy of implantation of a 3-bis(2chloroethyl)-1-nitrosourea (BCNU)-loaded wafer and intracarotid perfusion of BCNU-loaded nanoparticles for glioma treatment in vivo. BCNU-loaded poly(D,L-lactic acid) (PLA) nanoparticles coated with transferrin (Tf) were prepared by a solvent evaporation/diffusion method using $\mathrm{Tf}$ as the emulsifier. X-ray photoelectron spectroscopy, BrattonMarshall colorimetric assay and zeta-potential analysis confirmed the existence of $\mathrm{Tf}$ on the nanoparticles and their functional activities. BCNU-loaded PLA wafers were made of BCNU-loaded PLA microspheres. In vitro drug release behavior demonstrated that BCNU was released from the Tf-PLA nanoparticles and wafers in two distinct phases. The biodistribution of Tf-coated nanoparticles investigated by ${ }^{99 \mathrm{~m}} \mathrm{Tc}$-labeled single-photon emission computed tomography (SPECT) showed that the surface-containing Tf-PLA nanoparticles were concentrated in the brain. Inhibition of tumor growth in the C6 glioma-bearing animal model showed that combinational chemotherapy of BCNU-loaded wafer and BCNU-loaded PLA nanoparticles had a stronger inhibitory
\end{abstract}

Correspondence to: Dr Xubo Yuan, School of Materials Science and Engineering, Tianjin University, Tianjin 300070, P.R. China E-mail: xbyuan@tju.edu.cn

Dr Chunsheng Kang, Laboratory of Neuro-Oncology, Tianjin Neurological Institute, Tianjin Medical University General Hospital, Tianjin 300052, P.R. China

E-mail: kang97061@yahoo.com

*Contributed equally

Key words: glioma, nanoparticles, transferrin, poly(D,L-lactic acid), wafer, BCNU effect and prolonged the average survival time of rats (164\%) compared with that of the control group. Furthermore, the tumors of this treatment group were not visible by examination at 4 weeks. The results of this study demonstrate for the first time that combination therapy of implantation of a BCNUloaded wafer and intracarotid perfusion of BCNU-loaded nanoparticles may be a new strategy for glioma gene therapy.

\section{Introduction}

The treatment of glioma is one of the most difficult challenges in oncology. Despite treatment combining surgical resection, radiotherapy and chemotherapy, the 1-year median survival span of patients has not been significantly improved for 30 years $(1,2)$. The failure of chemotherapy is due to the inability of intravenously administered anticancer agents to reach the brain parenchyma. An endothelial cell monolayer associated with pericytes and astrocytes, known as the blood-brain barrier (BBB), separates the blood from the cerebral parenchyma and prevents the penetration of drugs into the central nervous system (CNS) $(3,4)$. In addition, the deficiency in pinocytic vesicles and the high metabolic capacity of cerebral endothelial cells limit the entrance of anticancer agents from the plasma to the CNS (5).

Various (neurosurgical) invasive strategies have been developed to improve the penetration of drugs into the brain. Among them, interstitial drug delivery was widely used for many years. Therapeutic agents were directly injected into the cerebral parenchyma. The implantation of polymeric matrices, such as microspheres loaded with the anticancer agent, has been used for the treatment of malignant gliomas (6-9). After implantation, the matrix releases the drug by hydrolytic degradation of the polymer. The Gliadels ${ }^{\circledR}$ wafer, another intracavitary treatment using a controlled release system, has also been developed (10). The therapeutic effect of the Gliadels wafer was tested in two phase III clinical trials $(11,12)$. The median survival time obtained in the treated patients was significantly prolonged in comparison to untreated patients, but the improvement was 


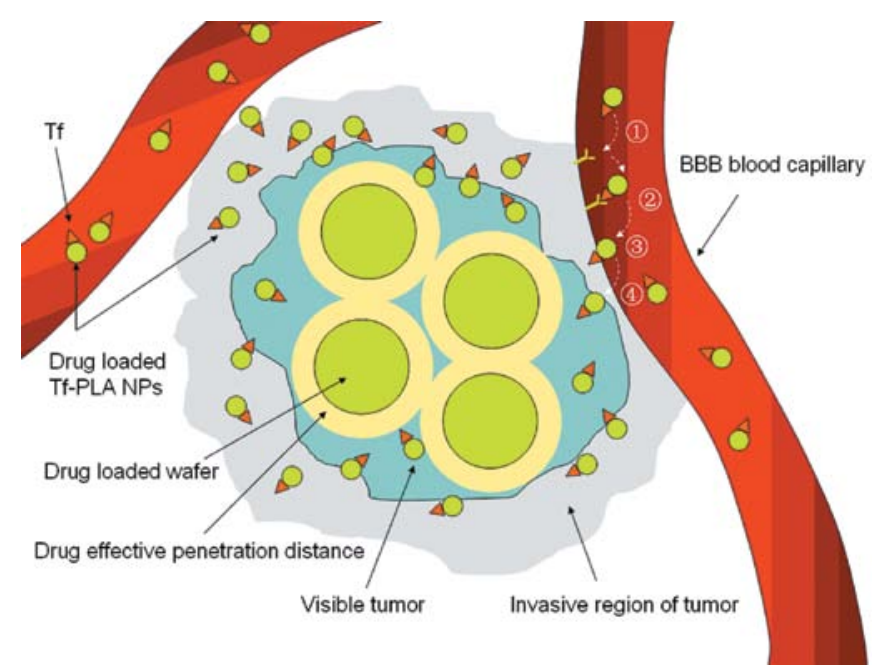

Figure 1. Schematic diagram of the treatment of glioma by the combination of BCNU-loaded PLA wafer implantation and intracarotid perfusion of the Tf-decorated BCNU-loaded PLA NPs. The wafer implanted in the visible region of the tumor, and the drug penetrated within a short distance from the wafer. The Tf-decorated NPs penetrated across the BBB blood capillary through four steps. 1, Approach to the surface of capillary well; 2, binding with the Tf receptor on the surface of capillary well; 3 , perfusion across the capillary well; 4 , diffusion into the glioma.

still limited since only a 2 -month increase in survival time could be obtained. Indeed, these invasive strategies rely on the diffusion of therapeutic molecules but this mechanism is poor in the cerebral parenchyma, as the diffusion rate of the drug decreases with the square of the diffusion distance (13). As a result, the chemotherapeutic agent may have an effect only at the vicinity of the implantation site (14), leading to failure of effective inhibition in the invasive region of the glioma (15).

Non-surgical strategies have also been investigated. Over the last decades, the most promising approach seems to be nanocarriers technology (16-18). Several major features of nanoparticles (NPs) make them especially useful for this application. Firstly, they represent an injectable drug delivery system with a particle size of $100 \mathrm{~nm}$ or less. These particles can easily enter brain capillaries and reach the surface of the brain microvascular endothelial cells (BMVEC) which form the BBB, and can even reach a deeper distance from the vascular surface (19). Secondly, NPs can be modified with specific targeting ligands that recognize the receptors on the BMVEC and mediate the NPs across the BBB. In particular, Pardridge proposed that insulin and transferrin (Tf) receptors are good candidates for this purpose (20). Third, following the delivery of the NPs to the disease site in the body, the polymer matrix can slowly degrade resulting in sustained release of the encapsulated therapeutic agents (21). Thus, the NPs have a dual capacity as drug carriers of targeting and sustained release capability.

Physiologically, the drug released from the implant wafer or microspheres may adopt a distribution pattern different from that released form the NPs injected. As shown in Fig. 1, drugs are released from the wafer at the center of the tumor to the periphery. The drug diffusion is limited to the vicinity of the implantation site, thus, it is inaccessible to the invasive regions of the tumor. However, the NPs via vascular injection could disperse inside the tumor from the periphery, and treat the tumor part that is far from the wafer location.

Based on these deductions, the objective of the present study was to evaluate the inhibition of glioma growth in vivo by combining the interstitial chemotherapy and the targeting drug delivery strategy. The BCNU-loaded wafers were implanted in the tumor while the BCNU-loaded PLA NPs decorated with Tf were administrated by intracarotid perfusion. The results showed that the combined therapy significantly prolonged the survival time of glioma-bearing rats in comparison with either treatment alone.

\section{Materials and methods}

Materials and reagents. Poly(D,L-lactic acid) (PLA, average molecular weight of $30 \mathrm{kDa}$ ) and transferrin (Tf) were purchased from Sigma. 3-bis(2-chloroethyl)-1-nitrosourea (BCNU) was obtained from the Tianjin Pharmaceutical Group Corporation. Diethylenetriaminepentaacetic acid anhydride (DTPAA) was obtained from Fluka. Sprague-Dawley (SD) rats were purchased from the Animal Center of the Chinese Military Medical Science Institute (Beijing, China). The Jiangwan animal stereotactic device model I C was from the Shanghai Second Military University (Shanghai, China).

Preparation of BCNU-loaded Tf-PLA NPs. BCNU-loaded Tf-PLA NPs were prepared according to a procedure described by our laboratory (22). Briefly, $20 \mathrm{mg}$ PLA was dissolved in $2 \mathrm{ml}$ acetone, followed by addition of $20 \mathrm{mg}$ BCNU with light prevention at $4{ }^{\circ} \mathrm{C}$. The resulting solution was poured to $25 \mathrm{ml}$ aqueous $\mathrm{Tf}(4 \mathrm{mg} / \mathrm{ml})$ in acetic acid-sodium acetate buffer $(0.1 \mathrm{M}, \mathrm{pH} 4.5)$ followed by reduced pressure rotary evaporation. The system was then stirred magnetically at room temperature until complete evaporation of the organic solvent. The NPs were purified on a Sephadex G50 column with $0.05 \mathrm{M}$ PBS to remove the uncoated Tf. The suspension was lyophilized until use.

Characterization of BCNU-loaded Tf-PLA NPs. The binding of Tf to PLA nanoparticles was confirmed by X-ray photoelectron spectroscopy (XPS, Perkin-Elmer PHI1600 ESCA system) and Zeta-potential measurements (BI-zeta plus; Brookhaven Instruments Co., USA). The morphology of NPs was observed using a JEOL-100CXII TEM. The particle size distribution were assessed by Photo Correlation Spectroscopy (PCS, 90 Plus/BI-MAS Particle size analyzer, USA).

Determination of BCNU loading efficiency. According to a procedure previously described, the Bratton-Marshall colorimetric assay was used to determine the BCNU loading (23). BCNU-loaded PLA NPs (10 mg) were dissolved in $500 \mu \mathrm{l}$ methylene chloride and then adjusted to $10 \mathrm{ml}$ with ethanol to precipitate the polymer. The suspension was centrifuged at $15,000 \mathrm{rpm}$ for $20 \mathrm{~min}\left(4^{\circ} \mathrm{C}\right)$. The supernatant $(200 \mu \mathrm{l})$ was added to $1 \mathrm{ml}$ of $0.5 \%$ sulfanilamide in $2 \mathrm{~N} \mathrm{HCl}$ and $1.8 \mathrm{ml}$ of deionized water. The sample was placed in a water bath at $50^{\circ} \mathrm{C}$ for $45 \mathrm{~min}$. After incubation, the sample was rapidly cooled in an ice bath. Thereafter, $100 \mu 1$ of N-(1-naphthyl) ethylenediamine dihydrochloride aqueous solution $(3 \mathrm{mg} / \mathrm{ml})$ were added. After $10 \mathrm{~min}$, the absorbance at $540 \mathrm{~nm}$ was measured. 
The drug loading efficiency were defined by the following expression:

Drug loading $(\% \mathrm{w} / \mathrm{w})=\frac{\text { Mass of drug in nanoparticles } \mathrm{x} 100}{\text { Mass of nanoparticles recovered }}$

In vitro drug release study. Release of BCNU from PLA NPs was investigated in vitro according to the procedure described by our laboratory (22). A known amount of BCNU-loaded NPs were placed in dialysis bags, which were incubated in vials containing phosphate-buffered saline (PBS, pH 7.4). The vials were placed in a thermostatic vibrator at $37^{\circ} \mathrm{C}$. Periodically, the PBS was removed and replaced with fresh PBS. The amount of BCNU released into PBS was calculated by the amount of $\mathrm{BCNU}$ remaining in the NPs because BCNU is unstable in the test condition. The solution was immediately determined for the presence of $\mathrm{BCNU}$ by the Bratton-Marshall colorimetric assay as above. The BCNU released into PBS per time point was divided by the total BCNU loaded and integrated over time to describe the cumulative release profile.

Preparation of BCNU loaded PLA wafer and drug release in vitro. BCNU-loaded PLA wafer was made from BCNUloaded PLA microparticles. BCNU-loaded PLA microparticles were prepared using a spray-drying method. Briefly, PLA (3\%) and BCNU (5\% of PLA) were co-dissolved in solvent ethyl acetate at $4^{\circ} \mathrm{C}$. The solution was spray-dried by a fluidized bed coater (Spray-dryer L-117, LaiHeng, Beijing) under the conditions of $7 \pm 2 \mathrm{ml} / \mathrm{min}$ of pump speed, $30 / \pm 4$ psi of spraying air pressure, and $40 \pm / 2{ }^{\circ} \mathrm{C}$ of inlet air temperature. The spray-dried microparticles were freeze-dried at 35 mTorr, and at $-50^{\circ} \mathrm{C}$ for $48 \mathrm{~h}$. Cylindrical wafers with $5 \mathrm{mg}$ weight ( $3 \mathrm{~mm}$ in diameter, $0.5 \mathrm{~mm}$ in thickness) were then prepared using the steel molding press. The wafers were stored at $4^{\circ} \mathrm{C}$ and sterilized by $\gamma$-rays before use. The in vitro drug release kinetics of the wafer were accessed using the same procedure employed by the BCNU-loaded NPs.

\section{Biodistribution of NPs in vivo}

Animal model. The C6 glioma-bearing animal model was developed as previously described (24). Adult male SD rats weighing between 200-250 g were anesthetized by an intraperitoneal injection of $10 \%$ chloral hydrate $(300 \mathrm{mg} / \mathrm{kg})$, and then fixed in a stereotactic apparatus. A $1.2-\mathrm{mm}$ burr hole was drilled into the right side of the skull ( $1 \mathrm{~mm}$ anterior and $3.0-3.5 \mathrm{~mm}$ lateral to the bregma) to expose the dura. Using a microliter syringe equipped with a 26 -gauge needle connected to the manipulating arm of the stereotactic apparatus, $1 \times 10^{6}$ of parental C6 glioma cells in 10- $\mu$ l serum-free DMEM were injected into the caudate nucleus at a depth of 4.0-4.5 mm from the dura over a 10-min period. The needle was left in place for $10 \mathrm{~min}$ and then slowly withdrawn. The burr hole was filled with bone wax and the scalp wound was closed with silk thread.

Radiolabeling and biodistribution of nanoparticles by single-photon emission computed tomography (SPECT). The radiolabeling and biodistribution of NPs was performed using the method reported by our laboratory (25). NPs (50 mg) were dispersed in $0.1-\mathrm{M}$ sodium phosphate, $0.1 \mathrm{M} \mathrm{NaCl}$ buffer ( $\mathrm{pH} 8.0$ ), followed by addition of $6 \mathrm{mg}$ DTPA in solid form. The mixture was stirred for $60 \mathrm{~min}$ at room temperature, and then centrifuged at $18,000 \mathrm{rpm}$ for $10 \mathrm{~min}$ followed by washing three times with buffer under sonication conditions. The NPs were resuspended in acetate buffer (0.1 M, pH 5.6) by sonication, and $0.3 \mathrm{ml}{ }^{99 \mathrm{~m}} \mathrm{Tc}(5.2 \mathrm{mCi})$ in saline, $0.5 \mathrm{ml}$ $\mathrm{SnCl}_{2}$ in hydrochloric acid solution $(0.01 \mathrm{M})$ was added. The mixture was stirred gently and was allowed to stand at room temperature for $60 \mathrm{~min}$. The labeled NPs were then purified by centrifugation at $18,000 \mathrm{rpm}$ for $10 \mathrm{~min}$, followed by sonication-washing three times with normal saline.

The biodistribution of ${ }^{99 \mathrm{~m}} \mathrm{Tc}-\mathrm{BSA}-\mathrm{PLA}$ and ${ }^{99 \mathrm{~m}} \mathrm{Tc}-\mathrm{Tf}-\mathrm{PLA}$ NPs were studied in C6 glioma-bearing SD rats (6-8-week-old, male) by SPECT (GE Discovery-VH, USA) image analysis. After injection of $100 \mu 1{ }^{99 \mathrm{~m}}$ Tc-BSA-PLA and ${ }^{99 \mathrm{~m}}$ Tc-Tf-PLA NPs solution $(1 \mathrm{mg} / \mathrm{ml})$ via the carotid artery, the anesthetized rats were placed ventrally on a platform for $2 \mathrm{~h}$. After a 2-h perfusion, $0.2 \mathrm{ml}$ of blood was collected. Then the rats were humanely sacrificed and the principle organs (brain, heart, liver, spleen, lung and kidney) were removed and weighed. The radioactivity of ${ }^{99 \mathrm{~m}} \mathrm{Tc}$ in the blood and the organs was assessed using a $\gamma$-counter and the results are expressed as the percentage of dose administered that accumulated in each organ $(\% \mathrm{ID} / \mathrm{g})$. Each test was repeated four times.

Tumor inhibition in vivo. When the tumor volume reached $4 \pm 1 \mathrm{~mm}^{3}$ as demonstrated by MRI on day 5 following injection, C6 glioma-bearing SD rats were randomly divided into 6 groups with 10 rats per group. The animals in the BCNU and Tf-PLA-BCNU NPs treatment groups received a dose of $2 \mathrm{mg} / \mathrm{kg}$ BCNU into the left common carotid. The animals in the blank PLA NPs group received empty NPs equivalent to the NPs concentration that was administered to the rats in the Tf-PLA-BCNU NPs treatment group. The rats in the BCNU-PLA wafer treatment group received a wafer implant. The wafers were implanted following the procedure below. The surgical wounds were reopened and the wafer was placed into the tumor with the aid of an operating microscope. After ensuring hemostasis, the skin was closed with surgical staples. For the evaluation of the effect of combinational therapy, the animals received a wafer implantation and BCNU-loaded Tf-PLA NPs equivalent to the NPs concentration that was administered to the rats of the Tf-PLA-BCNU NPs groups. The rats without treatment served as control.

The general behavior and survival of the rats in each group were observed. The enhanced MRI with Gd-DTPA (gadolium-diethylenetriamine pentaacetic acid) was used for monitoring the tumor size at different periods in the same animal and for comparison of the development of tumors in different groups of rats. The performance of MRI has been previously described (12). Whenever the rats died naturally or were sacrificed at one of the various time points, their brains were removed.

Statistical analyses. A commercially available software package SPSS10.0 was used for statistical analysis. The one-way analysis of variance (ANOVA) test was used to analyze the significance between the groups. The LSD (Least 

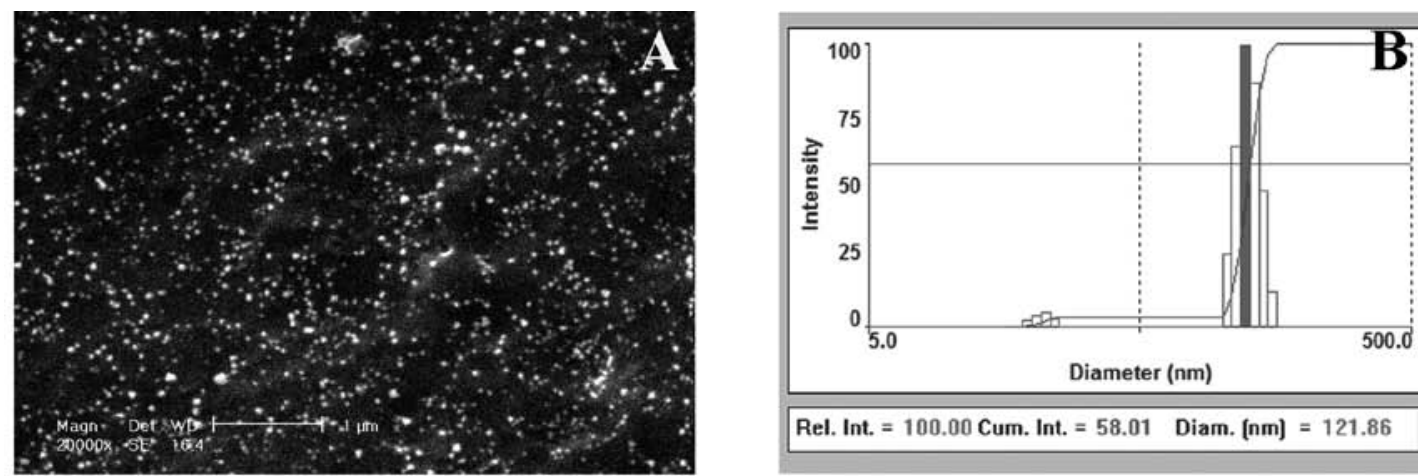

Rel. Int. $=100.00$ Cum. Int. $=58.01$ Diam. $[\mathrm{nm}]=121.86$
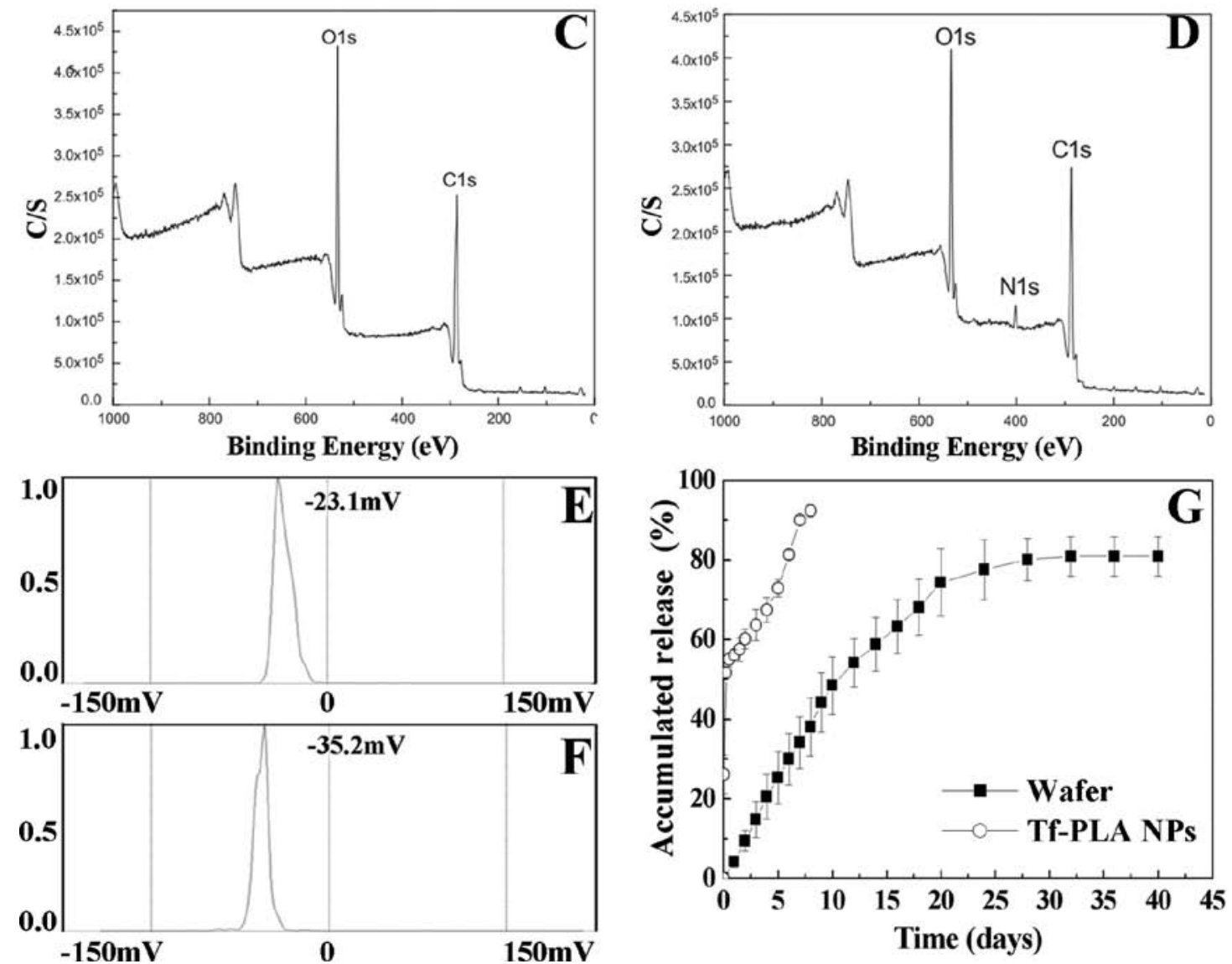

Figure 2. Construction and characterization of BCNU-loaded Tf-PLA NPs. (A) TEM images of BCNU-loaded Tf-PLA NPs. Scale bars, $1 \mu \mathrm{m}$. (B) The size and size distribution of BCNU-loaded Tf-PLA nanoparticles. High-resolution XPS spectra of BCNU-loaded PLA NPs (C) and BCNU-loaded Tf-PLA NPs (D). Zeta-potential analysis of BCNU-loaded PLA NPs (E) and BCNU-loaded Tf-PLA NPs (F). (G) In vitro release profile of BCNU from Tf-PLA NPs nanoparticles and wafers.

Significant Difference) multiple comparison test with different groups was used when the probability for ANOVA was statistically significant. Statistical significance was determined when $\mathrm{p}<0.05$.

\section{Results}

$B C N U$-carrier properties and drug release. Due to the emulsification of protein, the BCNU-loaded Tf-PLA NPs possessed a spherical shape (Fig. 2A) with an average size of $120 \pm 26 \mathrm{~nm}$ with a polydispersity index of 0.098 (Fig. 2B). Intact BCNU in the NPs was quantified via a colorimetric assay using the Bratton-Marshall assay, which is sensitive only to the whole
BCNU molecule. The drug content was only $3.87 \pm 0.52 \%$ because of the leakage of the BCNU from the NPs during the solvent evaporation process. The existence of the $\mathrm{Tf}$ protein on the surface of PLA NPs was proven by XPS and zeta-potential measurement. To exclude the possible nitrogen pollution from the atmosphere, PLA NPs prepared in PVA solution were used as a control sample. The XPS survey scans for the PLA and Tf-PLA NPs samples are shown in Fig. 2C and D. The enrichment of the surface with nitrogen-containing functionalities was evident for the Tf-coated samples, and the atomic percent content of nitrogen was $6.6 \%$, which was much larger than that on the PLA sample, where the atomic percent content of nitrogen was $<0.2 \%$. At the same time, the zeta-potential of 

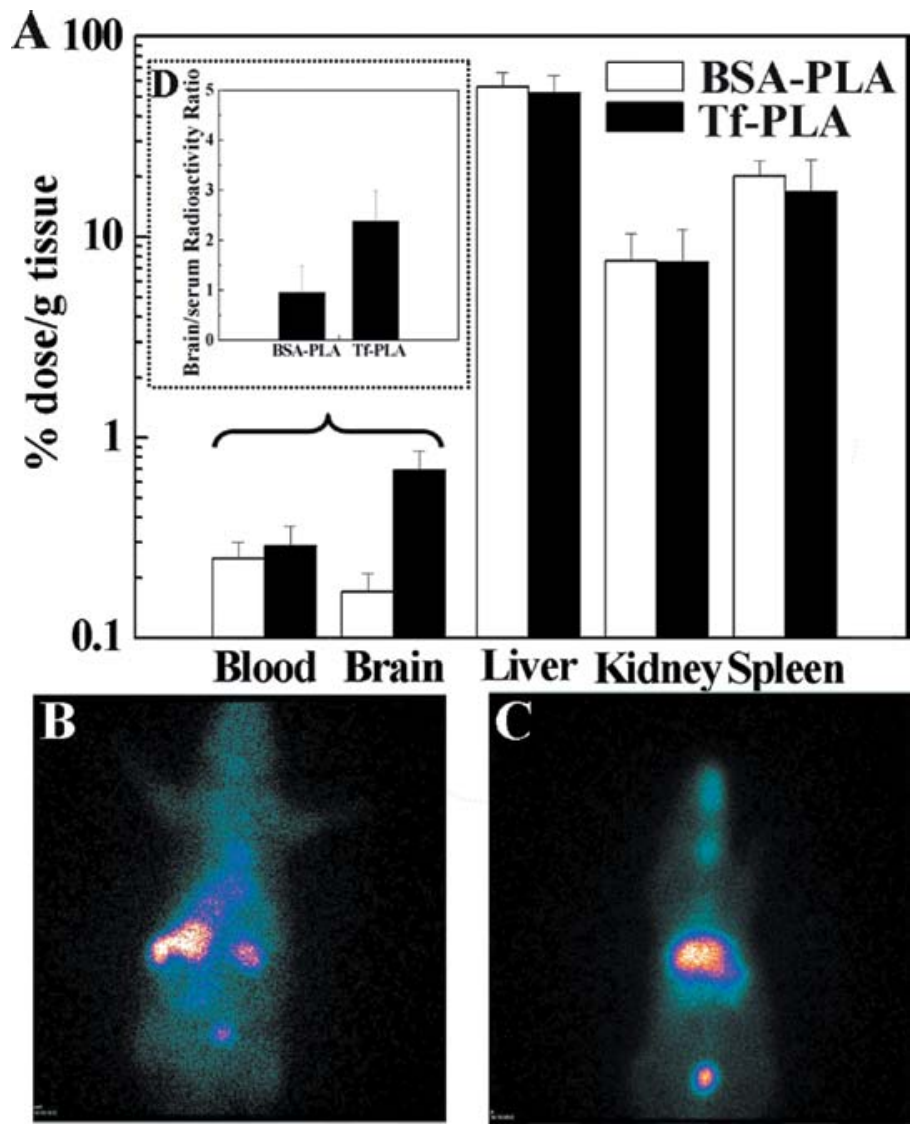

Figure 3. Biodistribution assay in vivo by the SPECT method. (A) Biodistribution of ${ }^{99 \mathrm{~m}} \mathrm{Tc}-\mathrm{BSA}-\mathrm{PLA}$ and ${ }^{99 \mathrm{~m}} \mathrm{Tc}-\mathrm{Tf}-\mathrm{PLA}$ NPs in the body of SD rats (n=4). Images of the rats injected with (B) ${ }^{99 \mathrm{~m}}$ Tc-BSA-PLA and (C) ${ }^{99 \mathrm{~m}}$ Tc-Tf-PLA NPs measured by SPECT. (D) Brain/plasma radioactivity ratios for the ${ }^{99 \mathrm{~m}} \mathrm{Tc}-\mathrm{BSA}$ PLA and ${ }^{99 \mathrm{~m}}$ Tc-Tf-PLA NPs.

the Tf-PLA NPs was $-23.1 \pm 3.4 \mathrm{mV}$ (Fig. 2E), which was higher than that $(-35.2 \pm 2.7 \mathrm{mV})$ of the native PLA NPs (Fig. 2F). This characterization demonstrated that Tf-PLA, a nanoscale drug delivery system with targeting function, had been successfully constructed.

BCNU was released from the Tf-PLA NPs and wafers in two distinct phases. Approximately $50 \%$ of the BCNU from Tf-PLA NPs was released during the initial $12 \mathrm{~h}$. This initial burst was followed by a much slower release of the majority of the remaining contents during the next 7 days. The size of the drug-loaded wafers were $3 \mathrm{~mm}$ in diameter and $0.5 \mathrm{~mm}$ in thickness with a drug content of $4.59 \%$. The Tf-PLA wafer released BCNU with nearly a zero-degree release in vitro for over 1 month with an accumulated drug release of $82 \%$. Due to the BCNU instability, it is impossible to achieve $100 \%$ drug release to PBS.

Biodistribution of Tf-PLA NPS. The biodistribution of radiolabeled Tf-PLA NPs in glioma-bearing rats after $2 \mathrm{~h}$ of intracarotid perfusion is shown in Fig. 3A. For both of the Tf-PLA and BSA-PLA NPs, the highest levels of radioactivity were found in the liver and spleen. However, for Tf-PLA NPs, the amount of the radioactivity in the brain was greatly increased compared to BSA-PLA NPs (Fig. 3B and C). This effect in the brain is further illustrated in Fig. 3D presenting brain/plasma radioactivity ratios for Tf-PLA and BSA-PLA NPs. This ratio for the Tf-PLA NPs increased by as much as 2.5-fold compared to BSA-PLA NPs $(\mathrm{p}<0.05)$.
Tumor inhibition growth in vivo. The general conditions of control and blank PLA NPs-treated rats were deteriorated 1 week after receiving the injection of parental C6 glioma cells and empty NPs. A reduction in drinking and eating, weight loss, and progressive left hemiplegia appeared. All the rats in the control and blank PLA NPs groups died within 15 days after implantation, giving a median survival time of $12.9 \pm 0.5$ days and $13.1 \pm 0.6$ days, respectively $(\mathrm{p}>0.05)$. Rats that received $\mathrm{BCNU}$ had a median survival time of $14.7 \pm 0.7$ days. There was no statistical difference in the survival time between the control and BCNU-treated groups $(\mathrm{p}=0.2340)$. In the BCNU-loaded Tf-PLA NPs treatment group, rats had similar general manifestations to those in the control group at the beginning of the second week after injection, but they had the smallest weight loss and lived longer with a median survival time of 19.7 days and an increased life-span (ILS) of $53 \%$. After 1-week treatment, 2 rats were at almost the same situation as rats in the control group, with clinical signs of hemiparalysis and bleeding eyes. The other 8 rats were in good health condition compared to the control groups. For two of these rats, a decrease in tumor volume was evident after 1 -week treatment. However, these animals died in the end due to tumor recurrence. The prolonged survival time in the BCNU-loaded Tf-PLA NPs treatment group indicated the significant difference as compared to the two control groups, the control and the BCNU-treated group $(p=0.001)$. Rats that received wafer treatment had a median survival time of $25.6 \pm 2.6$ days with an ILS of $98 \%$. One of these animals did 


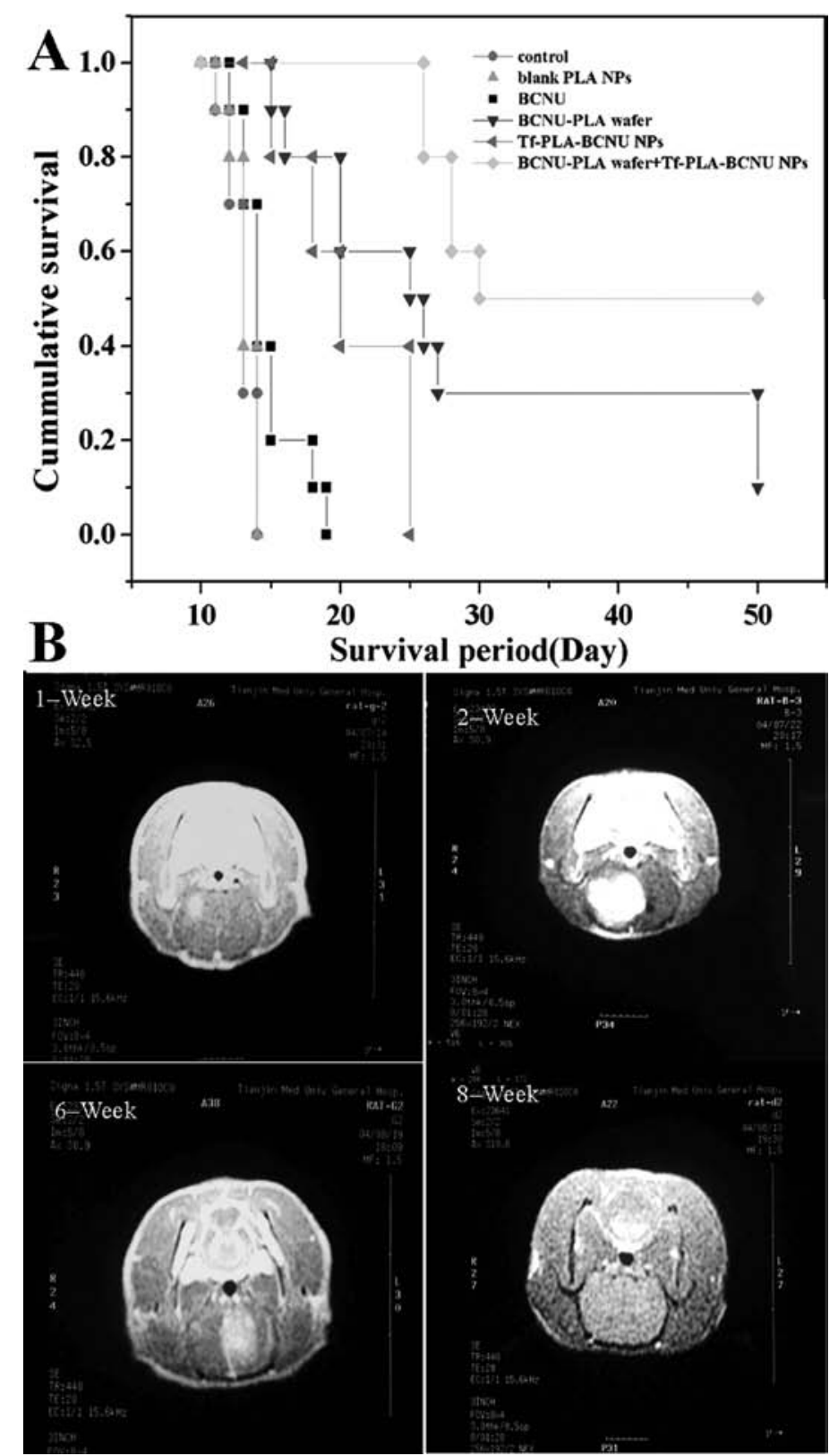

Figure 4. Antitumor effect in vivo. (A) Survival analysis in C6 control, free BCNU, blank PLA NPs, BCNU loaded PLA wafer, BCNU-loaded Tf-PLA NPs and BCNU loaded PLA wafer and BCNU-loaded Tf-PLA NPs combinational treatment groups. (B) A consecutive dynamic enhanced MRI study of a rat in BCNU-loaded PLA wafer and BCNU-loaded Tf-PLA NPs combinational treatment group.

not die as a result of brain-tumor growth. Rats that received wafer and Tf-PLA NPs treatment had a median survival time of $34.0 \pm 3.3$ days with an ILS of $164 \%$. Three of them were still alive and healthy 3 months after tumor treatment. The prolonged survival in the wafer and Tf-PLA NPs-treatment group indicated a significant difference as compared with the wafer groups and BCNU-loaded Tf-PLA NPs-treated group $(\mathrm{p}=0.001)$ (Fig. 4A).

In the rats of the control group and the treated group examined by MRI, the tumor foci began to develop on day 5 after implantation and appeared as enhanced distinct tumor foci on day 7. The foci grew rapidly and enlarged to occupy the largest portion of the right cerebral hemisphere at the end of the second week after implantation. They were homogeneously enhanced or irregularly rim-enhanced. Among the rats of the BCNU treated group examined by MRI, the tumor growth pattern was similar to those detected in the control and blank PLA NPs-treated groups. The tumor growth in 10 rats was slower than those in the control group. The MRI features of the rats in the wafer and Tf-PLA NPs treatment groups were the same as those in the control and blank PLA NPs treatment groups during the first weeks after implantation. Tumor foci were regressed significantly at 2 weeks and disappeared on MRI examination 4 weeks after implantation in 2 rats (Fig. 4B).

\section{Discussion}

The treatment of glioma is one of the most difficult challenges due to its infiltrative and aggressive nature. Various invasive strategies have been developed to improve the penetration of 
drugs into the brain (3). Among them, the wafer, another intracavitary treatment, has also been developed (26). The median survival time of the wafer-treated patients was significantly prolonged by 2 months in comparison with the untreated patients. However, this chemotherapeutic method may be effective only in the vicinity of the implantation site rather than the invasive region of the glioma (27). Over the last decades, the most promising approach seems to be nanotechnology. Following the NPs arrival at the disease site in the body, the polymer matrix slowly degrades resulting in sustained release of the encapsulated therapeutic agents. Thus, the NPs have a dual capacity of drug targeting delivery and sustained release capability $(28,29)$.

Glioma therapy via NPs has two remaining challenges due to the impermeable nature of the BBB and the lack of tumortissue specificity. Different strategies have been proposed to construct NPs that may cross the BBB and demonstrate specific delivery of the drug to the glioma. The binding of the endogenous and chimeric ligands renders the NPs capable of selectively recognizing brain capillary endothelial cells and cerebral tumoral cells, improving both the selective brain targeting and the tumor uptake of therapeutic molecules (30). Different ligands have been used to functionalize the nanoparticles, include Tf (31), RGD (32) and folic acid (33). Tf has been used as a tumor-targeting ligand for several drug delivery systems to the brain (34-37). Thus, in the present study, Tf was chosen as the biofactor to functionalize the BCNU-loaded PLA NPs for the targeting drug delivery. Although there have been various ligand conjugation strategies, the functionalization of drug-loaded PLA NPs is still a challenge, not only due to the absence of reactive groups in the PLA backbone chain but also due to the fact that inappropriate conjugation will lead to leakage of the entrapped drug (38). One of the methods for PLA NPs preparation is emulsion-solvent evaporation using serum albumin as a colloidal stabilizer $(39,40)$. The conformational immunochemistry study demonstrated that $90 \%$ of the serum albumin conjugated with PLA NPs can be considered as native (41). Accordingly, in our articles, Tf was successfully conjugated on the surface of PLA NPs via this method. The binding of Tf on the surface of PLA NPs was confirmed by XPS and zeta-potentials analysis. The zetapotentials of the NPs are negative, which is very important for obtaining low toxicity towards BBB (42). The characterization above demonstrated that the nanoscale drug delivery system with the targeting and transmembrane functions, Tf-PLA, was successfully constructed.

The targeting and transmembrane functions of PLA NPs in vivo were further analyzed by the radiotracer labeling and SPECT method. The results demonstrated that $\mathrm{Tf}$ could enhance BCNU-loaded PLA NPs intake in the brain. This method, however, could not give an answer whether the NPs get across the BBB and penetrate into the brain. From a physiological standpoint, it can be imaged that the NPs, at least part of them, adopt an 'outside to inside' method, penetrating across BBB and accumulating in the glioma, whether by passive diffusion or receptor-ligand binding induced endocytosis. There have been a number of reports suggesting that the integrity of $\mathrm{BBB}$ in the brain tumor is compromised by the angiogenic processes in tumors. These new vessels are leaky and thus allow for increased brain penetration of molecules
$(43,44)$. There are also studies reporting that an increase in vessel wall thickness is a common feature of the glioma vasculature and is attributed to endothelial cell hyperplasia, leading to an increase in non-selective transendothelial transport. These changes may contribute to the transport of NPs to the brain tumor across the BBB. In addition to the passive diffusive method, the receptor-mediated method is the most effective way for the NPs to penetrate the BBB.

In the present study, although only a few NPs were accumulated in the brain, the antitumor effect of BCNU-loaded Tf-PLA NPs was still pronounced. On the other hand, although the wafer treatment is much better than BCNU-loaded Tf-PLA NPs, the limitation in the efficacy of the wafer may be attributed to the rapid elimination of BCNU. It has been shown that the penetration distance was $\sim 5 \mathrm{~mm}$ at day 1 and $\sim 1 \mathrm{~mm}$ at day 3. The enhanced penetration observed on day 1 appears to be due to accumulation of extracellular fluid caused by transient, vasogenic edema, which disappeared by day 3. Accordingly, the most optimal efficacy seems to be acquired by wafer and NPs interstitial chemotherapy. Therefore, in this study, as a compensation for the wafer interstitial chemotherapy, BCNUloaded Tf-PLA NPs were administrated via intracarotid perfusion. The results demonstrated the hypothesis, as shown in Fig. 1, that combination of wafer interstitial chemotherapy and intracarotid perfusion of functionalized BCNU-loaded nanoparticles could significantly prolong the survival ratio of the animal model, suggesting the potential clinical significance of this method.

\section{Acknowledgements}

This study was financially supported by the National Natural Science Foundation of China (Grant no. 30971136, 51073118, 81001128, 51103107) and the Program for New Century Excellent Talents in University (NCET-08-0393).

\section{References}

1. Jiang G, Wei ZP, Pei DS, Xin Y, Liu YQ and Zheng JN: A novel approach to overcome temozolomide resistance in glioma and melanoma: inactivation of MGMT by gene therapy. Biochem Biophys Res Commun 406: 311-314, 2011.

2. Quick A, Patel D, Hadziahmetovic M, Chakravarti A and Mehta M: Current therapeutic paradigms in glioblastoma. Rev Recent Clin Trials 5: 14-27, 2010.

3. Harn HJ, Lin SZ, Lin PC, Liu CY, Liu PY, Chang LF, Yen SY, Hsieh DK, Liu FC, Tai DF and Chiou TW: Local interstitial delivery of z-butylidenephthalide by polymer wafers against malignant human gliomas. Neuro Oncol 13: 635-648, 2011.

4. Wang X, Duan X, Yang G, Zhang X, Deng L, Zheng H, Deng C, Wen J, Wang N, Peng C, Zhao X, Wei Y and Chen L: Honokiol crosses $\mathrm{BBB}$ and $\mathrm{BCSFB}$, and inhibits brain tumor growth in rat 9L intracerebral gliosarcoma model and human U251 xenograft glioma model. PLoS One 6: e18490, 2011.

5. Reese TS and Karnovsky MJ: Fine structural localization of a blood-brain barrier to exogenous peroxidase. J Cell Biol 34: 207-217, 1967.

6. Lesniak MS, Upadhyay U, Goodwin R, Tyler B and Brem H: Local delivery of doxorubicin for the treatment of malignant brain tumors in rats. Anticancer Res 25: 3825-3831, 2005.

7. Nakabayashi H, Sakaguchi M, Katsuyama J and Hakuba A: Proliferative potential of meningiomas evaluated by proliferating cell nuclear antigen expression. J Neurooncol 24: 209-217, 1995.

8. DiMeco F, Li KW, Tyler BM, Wolf AS, Brem H and Olivi A: Local delivery of mitoxantrone for the treatment of malignant brain tumors in rats. J Neurosurg 97: 1173-1178, 2002. 
9. Menei P, Venier MC, Gamelin E, Saint-André JP, Hayek G, Jadaud E, Fournier D, Mercier P, Guy G and Benoit JP: Local and sustained delivery of 5-fluorouracil from biodegradable microspheres for the radiosensitization of glioblastoma: a pilot study. Cancer 86: 325-330, 1999.

10. Westphal M, Ram Z, Riddle V, Hilt D and Bortey E: Executive Committee of the Gliadel Study Group. Gliadel wafer in initial surgery for malignant glioma: long-term follow-up of a multicenter controlled trial. Acta Neurochir (Wien) 148: 269-275, 2006.

11. Brem H, Piantadosi S, Burger PC, Walker M, Selker R, Vick NA, Black K, Sisti M, Brem S and Mohr G: Placebo-controlled trial of safety and efficacy of intraoperative controlled delivery by biodegradable polymers of chemotherapy for recurrent gliomas. The Polymer-brain Tumor Treatment Group. Lancet 345: 1008-1012, 1995.

12. Westphal M, Hilt DC, Bortey E, Delavault P, Olivares R, Warnke PC, Whittle IR, Jääskeläinen J and Ram Z: A phase 3 trial of local chemotherapy with biodegradable carmustine (BCNU) wafers (Gliadel wafers) in patients with primary malignant glioma. Neuro Oncol 5: 79-88, 2003.

13. Pardridge WM: Non-invasive drug delivery to the human brain using endogenous blood-brain barrier transport systems. Pharm Sci Technolo Today 2: 49-59, 1999.

14. Balossier A, Dörner L, Emery E, Heese O, Mehdorn HM, Menei $\mathrm{P}$ and Singh $\mathrm{J}$ : Incorporating BCNU wafers into malignant glioma treatment: European case studies. Clin Drug Investig 30: 195-204, 2010.

15. Ogris M, Brunner S, Schüller S, Kircheis R and Wagner E: PEGylated DNA/transferrin-PEI complexes: reduced interaction with blood components, extended circulation in blood and potential for systemic gene delivery. Gene Ther 6: 595-605, 1999.

16. Béduneau A, Saulnier P and Benoit JP: Active targeting of brain tumors using nanocarriers. Biomaterials 28: 4947-4967, 2007.

17. Han L, Zhang AL, Wang HJ, Pu PY, Kang CS and Chang J: Tat-BMPs-PAMAM conjugates enhance therapeutic effect of small interference RNA on U251 glioma cells in vitro and in vivo. Hum Gene Ther 21: 417-426, 2010.

18. Tang R, Ji W, Panus D, Palumbo RN and Wang C: Block copolymer micelles with acid-labile ortho ester side-chains: synthesis, characterization, and enhanced drug delivery to human glioma cells. J Control Release 151: 18-27, 2011.

19. Han L, Zhang A, Wang $\mathrm{H}, \mathrm{Pu}$ P, Kang $\mathrm{C}$ and Chang $\mathrm{J}$ Construction of novel brain-targeting gene delivery system by natural magnetic nanoparticles. J Appl Pol Sci 121: 3446-3454, 2011.

20. Pardridge WM: Preparation of Trojan horse liposomes (THLs) for gene transfer across the blood-brain barrier. Cold Spring Harb Protoc 2010: pdb.prot5407, 2010.

21. Cinti C, Taranta M, Naldi I and Grimaldi S: Newly engineered magnetic erythrocytes for sustained and targeted delivery of anti-cancer therapeutic compounds. PLoS One 6: e17132, 2011.

22. Kang C, Yuan X, Zhong Y, Pu P, Guo Y, Albadany A, Yu S, Zhang Z, Li Y, Chang J and Sheng J: Growth inhibition agains intracranial C6 glioma cells by stereotactic delivery of BCNU by controlled release from poly(D,L-lactic acid) nanoparticles Technol Cancer Res Treat 8: 61-70, 2009.

23. Loo TL and Dion RL: Colorimetric method for the determination of 1,3-bis(2-chloroethyl)-1-nitrosourea. J Pharm Sci 54: 809-810, 1965.

24. Xu D, Jia Q, Li Y, Kang C and Pu P: Effects of Gamma Knife surgery on C6 glioma in combination with adenoviral p53 in vitro and in vivo. J Neurosurg 105 (Suppl): 208-213, 2006.

25. Yuan, XB, Li H and Yuan YB: Preparation of cholesterolmodified chitosan self-aggregated nanoparticles for delivery of drugs to ocular surface. Carbohyd Polym 65: 337-345, 2006.

26. Limentani SA, Asher A, Heafner M, Kim JW and Fraser R: A phase I trial of surgery, Gliadel wafer implantation, and immediate postoperative carboplatin in combination with radiation therapy for primary anaplastic astrocytoma or glioblastoma multiforme. J Neurooncol 3: 241-244, 2005.
27. Kircheis R, Wightman L, Schreiber A, Robitza B, Rössler V, Kursa $\mathrm{M}$ and Wagner E: Polyethylenimine/DNA complexes shielded by transferrin target gene expression to tumors after systemic application. Gene Ther 8: 28-40, 2001.

28. Passarella RJ, Spratt DE, van der Ende AE, Phillips JG, Wu H, Sathiyakumar V, Zhou L, Hallahan DE, Harth E and Diaz R: Targeted nanoparticles that deliver a sustained, specific release of Paclitaxel to irradiated tumors. Cancer Res 70: 4550-4559, 2010.

29. Ding H, Inoue S, Ljubimov AV, Patil R, Portilla-Arias J, Hu J, Konda B, Wawrowsky KA, Fujita M, Karabalin N, Sasaki T, Black KL, Holler E and Ljubimova JY: Inhibition of brain tumor growth by intravenous poly ( $\beta$-L-malic acid) nanobioconjugate with $\mathrm{pH}$-dependent drug release. Proc Natl Acad Sci USA 107: $18143-18148,2010$

30. Xin H, Jiang X, Gu J, Sha X, Chen L, Law K, Chen Y, Wang X, Jiang Y and Fang X: Angiopep-conjugated poly(ethylene glycol)co-poly(e-caprolactone) nanoparticles as dual-targeting drug delivery system for brain glioma. Biomaterials 32: 4293-4305, 2011.

31. Ren WH, Chang J, Yan CH, Qian XM, Long LX, He B, Yuan XB, Kang CS, Betbeder D, Sheng J and Pu PY: Development of transferrin functionalized poly(ethylene glycol)/poly(lactic acid) amphiphilic block copolymeric micelles as a potential delivery system targeting brain glioma. J Mater Sci Mater Med 21: 2673-2681, 2010.

32. Waite CL and Roth CM: PAMAM-RGD conjugates enhance siRNA delivery through a multicellular spheroid model of malignant glioma. Bioconjug Chem 20: 1908-1916, 2009.

33. Kang C, Yuan X, Li F, Pu P, Yu S, Shen C, Zhang Z and Zhang Y: Evaluation of folate-PAMAM for the delivery of antisense oligonucleotides to rat C6 glioma cells in vitro and in vivo. J Biomed Mater Res 93: 585-594, 2010

34. Li Y, Ogris M, Wagner E, Pelisek J and Rüffer M: Nanoparticles bearing polyethyleneglycol-coupled transferrin as gene carriers: preparation and in vitro evaluation. Int $\mathbf{J}$ Pharm 259: 93-101, 2003.

35. Soni V, Kohli DV and Jain SK: Transferrin coupled liposomes as drug delivery carriers for brain targeting of 5-florouracil. J Drug Target 13: 245-250, 2005.

36. Eavarone DA, Yu X and Bellamkonda RV: Targeted drug delivery to C6 glioma by transferrin-coupled liposomes. J Biomed Mater Res 51: 10-14, 2000

37. Hatakeyama H, Akita H, Maruyama K, Suhara $T$ and Harashima H: Factors governing the in vivo tissue uptake of transferrin-coupled polyethylene glycol liposomes in vivo. Int J Pharm 281: 25-33, 2004 .

38. Madhavan Nampoothiri K, Nair NR and John RP: An overview of the recent developments in polylactide (PLA) research. Bioresour Technol 101: 8493-8501, 2010.

39. Boury F, Ivanova T, Panaieotov I, Proust JE, Bois A and Richou J: Dilatational properties of adsorbed poly (D,L-lactide) and bovine serum albumin monolayers at the dichloromethane/ water interface. Langmuir 11: 1636-1644, 1995.

40. Boury F, Marchais H, Benoit JP and Proust JE: Surface characterization of poly(alpha-hydroxy acid) microspheres prepared by a solvent evaporation/extraction process. Biomaterials 18: 125-136, 1997.

41. Verrecchia T, Huve P, Bazile D, Veillard M, Spenlehauer G and Couvreur P: Adsorption/desorption of human serum albumin at the surface of poly(lactic acid) nanoparticles prepared by a solvent evaporation process. J Biomed Mater Res 27: 1019-1028, 1993.

42. Lockman PR, Koziara JM, Mumper RJ and Allen DD: Nanoparticle surface charges alter blood-brain barrier integrity and permeability. J Drug Target 12: 635-641, 2004.

43. Stewart DJ: A critique of the role of the blood-brain barrier in the chemotherapy of human brain tumors. J Neurooncol 20: 121-139, 1994.

44. van den Bent MJ: The role of chemotherapy in brain metastases. Eur J Cancer 39: 2114-2120, 2003. 